\title{
3 Research Square

\section{Differences in brain activity between fast and slow responses on psychomotor vigilance task: an fNIRS study.}

Mateus G. Nogueira

UFABC: Universidade Federal do ABC

Mateus Silvestrin

UFABC: Universidade Federal do ABC

Cândida S. F. Barreto

UFABC: Universidade Federal do ABC

João Ricardo Sato

UFABC: Universidade Federal do ABC

Rickson C. Mesquita

UNICAMP: Universidade Estadual de Campinas

Claudinei Biazoli

UFABC: Universidade Federal do ABC

Abrahão Fontes Baptista ( $\nabla$ a.baptista@ufabc.edu.br)

UFABC: Universidade Federal do ABC https://orcid.org/0000-0001-7870-3820

\section{Research Article}

Keywords: Sustained attention, psychomotor vigilance task, fNIRS

Posted Date: May 4th, 2021

DOl: https://doi.org/10.21203/rs.3.rs-387613/v1

License: (9) This work is licensed under a Creative Commons Attribution 4.0 International License. Read Full License

Version of Record: A version of this preprint was published at Brain Imaging and Behavior on January 29th, 2022. See the published version at https://doi.org/10.1007/s11682-021-00611-8. 


\section{Abstract}

Attention is a basic human function underlying every other cognitive process. It is demonstrated in the functional Magnetic Resonance Imaging literature that frontoparietal networks are involved with attentive performance while default mode networks are involved with inattentive performance. Yet, it is still not clear whether similar results would be found with functional Near-Infrared Spectroscopy. The goal of our study was to investigate differences in hemodynamic activity measured by functional Near-Infrared Spectroscopy between fast and slow responses on a simple sustained attention task both before and after stimulus onset. Thirty healthy adults took part in the study. Our results have shown differences between fast and slow responses only on channels over medial frontal cortex and inferior parietal cortex $(p<0,05)$. These differences were observed both before and after stimulus presentation. It is discussed that functional Near-Infrared Spectroscopy is a good tool to investigate the frontoparietal network and its relationship with performance on attention tasks; it could be used to further investigate other approaches on attention, such as the dual network model of cognitive control and brain states views based on complex systems analysis; and finally, it could be used to investigate attention on naturalistic settings.

\section{Introduction}

Sustained attention (SA) refers to the maintenance of engagement in a specific task for long periods of time (Fortenbaugh et al., 2017). It requires prolonged conscious effort of cognitive processing for at least ten seconds (Langner \& Eickhoff, 2013). Because of its monotonous characteristics, simple tasks such as detection or discrimination tasks impose more demand on SA than tasks that require complex stimulus processing or high order executive function (Langner \& Eickhoff, 2013). Both vigilance and tonic alertness can be considered synonyms for SA. The importance of SA lies in the proposition that it might be one of the earliest and simplest forms of "self-regulatory and endogenous control over one's attention and cognitive system" (Yu \& Smith, 2016).

The psychomotor vigilance task (PVT) is an example of a simple detection task that is used to assess SA. It was originally proposed by (Dinges \& Powell, 1985) as a tool to investigate the effects of sleep deprivation on performance during sustained operation. During this task, participants are asked to respond to a stimulus every time it occurs, and their reaction times (RT) are tracked. The task allows for quantitative measures such as RT means, RT variability, and RT decrement. Currently, adaptations for computers or hand devices have been made (Arsintescu et al., 2017; Khitrov et al., 2014), which allows the PVT to be employed in a variety of contexts. For example, researchers have employed functional magnetic resonance imaging ( $\mathrm{fMRI}$ ) during PVT to investigate brain patterns associated with autism and obsessive-compulsive disorder (Carlisi et al., 2017), post traumatic brain injury fatigue (Nordin et al., 2016), and neuropsychology evaluation on workers during night shifts (Jaipurkar et al., 2019; McHill \& Wright, 2019).

In one of the first studies applying $\mathrm{fMRI}$ to assess neural correlates of performance variation during PVT, Drummond et al. compared the hemodynamic activity related to fast and slow RT responses (Drummond 
et al., 2005). They found that fast RT was related to the right middle frontal gyrus, right inferior parietal lobe, premotor area (dorsal, and ventral - supplementary motor area), and subcortical regions (like putamen, caudate nucleus and globus pallidus). On the other hand, slow RT was related to activity on medial frontal cortices and the ventral anterior cingulate cortex. The results demonstrated involvement of the Frontoparietal Network (FPAN) (Lawrence et al., 2003; Sturm \& Willmes, 2001) during fast responses, and of the Default Mode Network (DMN) (Raichle \& Snyder, 2007) during slow responses.

The neural mechanisms of PVT-like tasks were further explored by Langner and Eickhoff in an extensive metanalysis that only included studies using simple detection and RT tasks (Langner \& Eickhoff, 2013). The authors found involvement of the same areas above mentioned, pointing to a right lateralized frontoparietal network for vigilance or sustained attention. They argue that each component of this network might have different functions that could be disentangled in bottom up and top down processes, resulting in subtypes of attentional processing.

Dosenbach et al. propose a similar approach to explain attention and cognitive control in their Dual Network approach (Dosenbach et al., 2007). The authors argue that any goal-directed cognitive task involves two brain networks that allow for different brain functions and, consequently, for different patterns of brain activation. An interplay between a dorsal anterior cingulate cortex/medial superior frontal cortex (dACC/msFC) network and a bilateral anterior insula/frontal operculum (al/fO) network would allow for (1) a time-locked activity relative to the beginning of task periods for control preparation or initiation of small task sets or trials, (2) a sustained activity responsible for maintaining engagement during longer periods, and (3) a performance-related activity responsible for self-monitoring and feedback processing.

This proposition leads to at least two important implications. First, it indicates that, when studying performance on cognitive tasks, one should look not only for the hemodynamic response and sustained block activities, but also to pre-stimulus activity. Indeed, it has been demonstrated that ongoing brain activity before stimulus presentation could predict participants' performance on an adapted Stroop task (Coste et al., 2011; Nozawa et al., 2014). Second, it is suggested that hemodynamic activity may present different characteristics depending on performance and on brain area (an example can be found on (Hinds et al., 2013).

The vast majority of evidence on the hemodynamics correlates of PVT comes from a single method, namely, functional magnetic resonance (fMRI). An alternative is found on functional Near-Infrared Spectroscopy (fNIRS), a neuroimaging modality that can measure cortical hemodynamics by the emission and detection of near-infrared light ( 700 - $900 \mathrm{~nm}$ ) (Len-Carrin \& Len-Domnguez, 2012). From a physiological perspective, fNIRS is analogous to fMRI since it measures hemodynamic changes, putatively proxying neural activity through neurovascular coupling mechanisms. In comparison with fMRI, fNIRS is less costly, easily handled and less sensitive to motion artifacts; it also allows for measurements at natural settings (i.e., outside a lab or a scanner). These advantageous features allow for higher applicability and for investigation in less artificial conditions, which have fostered the use of 
fNIRS in several studies on cognition. Examples of application of fNIRS on attention research can be found in (Harrivel et al., 2013), which employed fNIRS to discriminate between rest and attention tasks; and in (Bogler et al., 2014), which demonstrated that oxyhemoglobin changes measured by fNIRS correlate with RT variation in a Stroop task.

Regarding the use of fNIRS on investigation of brain correlates of PVT, only a few studies can be found. Hall et al. used PVT to investigate attentional deficits in adolescents that were born preterm (Hall et al., 2008); although differences on attention measures were found, no differences on fNIRS data were observed. Hiroyasu et al. demonstrated that there are hemodynamic differences between sensory modality (auditory or visual) of PVT and that there are also differences between PVT and a GO/NOGO sustained attention task (Hiroyasu et al., 2012). Borragán et al. applied PVT to study prefrontal connectivity and sleep deprivation in healthy young adults, and found that decreased brain connectivity mediates sleep impairment and sustained attention depletion (Borragán et al., 2019). Yet, no study directly compared differences in hemodynamic activity associated with good and poor performance on PVT.

Considering the relevance of PVT for the neuropsychology field and the possibility to investigate its neurophysiological correlates with a more accessible and flexible tool, in this work we aimed to first replicate (Drummond et al., 2005) findings using fNIRS, and then to further explore the advantages of fNIRS to test (Dosenbach et al., 2007) dual network model for sustained attention. Specifically, our objectives were to assess: (a) which areas, if any, present differences in the hemodynamic response between fast and slow RTs; (b) which areas, if any, present differences on variation of oxy- and deoxyhemoglobin levels before stimulus onset between fast and slow RTs, and; (c) potential differences on the shape of the hemodynamic curve between areas and between fast and slow responses. We hypothesized that there would be differences on both hemodynamic activity and pre-stimulus hemodynamic variation between fast and slow RTs, and that these differences would be observed primarily on frontoparietal areas. We also hypothesized that it would be possible to observe different shapes of the hemodynamic curve that resembles the different brain/cognitive functions proposed by Dosebanch et al. (2007).

\section{Materials And Methods}

Data and code are public and available at Mendeley Data (Nogueira, 2021).

\section{Participants}

Thirty healthy graduate and undergraduate students took part in the study. We aimed to increase the sample size used on Drummond et al. (2005) and Bogler et al. (2014) to avoid type-Il error. All participants were between 18 and 35 years old, right-handed, and with no history of mental illness, drug abuse, or use of psychoactive medications that could affect the hemodynamic signals. Five participants were excluded from the study due to bad quality signals on more than $20 \%$ of fNIRS channels. Therefore, twenty-five participants were included in data analysis ( $26 \pm 6$ years old), 11 females and 14 males. 


\section{Experimental Procedures}

\section{Psychomotor Vigilance Task (PVT).}

We used a computer version of the PVT, as proposed by Khitrov et al. (Khitrov et al., 2014). Participants comfortably seated in front of a computer screen presenting a black background, occasionally a red millisecond counter was presented. As soon as the counter appeared, the participant should click in the mouse, the counter would show and register the participants' reaction time (RT) and then would disappear. The timer appeared with an interstimulus interval (ISI) ranging from 10 to 20 seconds, randomly assigned from a uniform distribution. The task lasted 15 minutes with a total of approximately 60 trials.

\section{fNIRS acquisition}

While performing the PVT, participants had their heads scanned with an fNIRS device (NIRScout, NIRx Medical Technologies, Glenn Head, NY). The sources emitted light on two wavelengths, $760 \mathrm{~nm}$ and 850 $\mathrm{nm}$, at a sampling rate of $7.8 \mathrm{~Hz}$. A montage with 16 sources and 16 detectors was used, summing up 49 channels, with source-detection separation of three centimeters. The optodes were placed on a cap following the 10-20 EEG international system (Figure 2) (Klem et al., 1999). Since previous studies have shown that sustained attention is mainly a right lateralized function (Langner \& Eickhoff, 2013), optodes were only distributed on the right side of the scalp (See Supplementary Material 1 for illustration). fNIRS channels were grouped into eight regions of interest (ROIs) identified as (1) medial prefrontal cortex, (2) dorsolateral pre-frontal cortex, (3) lateral pre-frontal cortex, (4) pre-central gyrus, (5) post-central gyrus, (6) superior parietal cortex, (7) inferior parietal cortex, (8) occipital cortex (see Supplementary Material 2 for detailed information on channels and its corresponding ROIs). The software fOLD (Morais et al., 2018) was used to make sure that the ROls were properly covered by the optodes.

\section{Data Analysis}

\section{Behavioral data}

For all subjects, the RTs were measured on each trial. Participants' responses were classified according to Khitrov \& Laxminarayan (2014) proposition: RTs shorter than 100 ms were classified as anticipation, RTs longer than 500 ms were considered minor lapses, RTs longer than 1,000 ms were denominated major lapses, and RTs longer than 3,000 ms were considered no responses. Responses presenting RTs between 100 and $500 \mathrm{~ms}$ were considered regular responses. For each participant's regular responses, we calculated the longest and shortest RT deciles. The responses on the lowest decile were considered fast responses and those on the highest decile were considered slow responses. 


\section{fNIRS data}

Initially, we checked the raw signal quality using the software NirsLab (NIRx Medical Technologies, Glenn Head, NY). Briefly, the coefficient of variation (CV) was calculated for each channel. Channels that presented CV higher than $15 \%$ were considered bad channels and were excluded from the analysis. Data from participants that presented more than $20 \%$ of bad channels were also excluded.

Raw light intensity data from the remaining channels were transformed into oxy-hemoglobin $(\mathrm{HbO})$ and deoxy-hemoglobin $(\mathrm{Hb})$ concentration changes by applying the modified Beer-Lambert law (Cope \& Delpy, 1988). A pre-whitening filter was applied to remove data temporal autocorrelation. A band-pass filter from 0.01 to 0.2 was applied in order to decrease physiological artifacts. A General Linear Model (GLM) was performed for both $\mathrm{HbO}$ and $\mathrm{Hb}$ time series of each channel. In the $\mathrm{GLM}$, variations of $\mathrm{Hb}$ and $\mathrm{HbO}$ concentration levels at each channel were modeled by a canonical Hemodynamic Response Function (HRF) convolved with a binary time series containing the stimulus timings. The beta regression coefficients were obtained as an indicator of brain activity for each channel. Beta values were then compared between conditions for each individual using a t-test with a significance level of 0.05 . For data visualization, t-test results were plotted in statistical parametric mappings (SPM) comparing data relative to fast responses versus baseline, slow responses versus baseline and fast versus slow responses. To assess the correlation between brain activity and behavior, Spearman correlation tests were conducted between beta values and RT measures.

\section{Pre-stimulus activity and time course visualization}

Besides the standard SPM analysis, an alternative analysis was conducted on the same data set in R ( R Core Team, 2018). Briefly, raw data from light intensity collected by fNIRS channels were converted to optical densities on Homer2 v.2.3 (Huppert et al., 2009) following the pipeline from (Rodrigues et al., 2019). It was then converted to concentration variation of $\mathrm{HbO}$ or $\mathrm{Hb}$. A bandpass filter from 0.01 to 0.08 $\mathrm{Hz}$ was applied over the optical densities obtained after data collection, it was used in order to maintain only low frequency signals for analysis. These frequencies are shown to be more representative of spontaneous fluctuations in brain activity (Hosomi et al., 2019). Measures of optical densities were then converted to oxyhemoglobin $(\mathrm{HbO})$ and deoxyhemoglobin $(\mathrm{Hb})$ concentration changes using a modified Beer-Lambert Law. A principal component analysis (PCA) was computed and then the two main components were removed in order to remove the hemispheric signal, an approximation of the global signal removal.

For purposes of data visualization, the time series of oxyhemoglobin variation levels were averaged across participants for each channel and each condition. Since stimulus duration varied in a time frame of milliseconds, we considered that the stimulus presentation and its following response lasted for $1 \mathrm{sec}$. A window of $6 \mathrm{~s}$ before and $10 \mathrm{~s}$ after stimulus presentation was considered. For purposes of prestimulus data analysis, we considered only the signals related to three seconds before and two seconds after stimulus presentation for statistical analyses. Since hemodynamic response is slow, we considered 
that data around two seconds after stimulus presentation still referred to events occurring before it. Average oxyhemoglobin concentration was calculated on this period for each $\mathrm{ROI}$ in each condition for each participant. Wilcoxon signed rank tests were conducted to check the differences between conditions on each ROI. Bonferroni corrections were conducted to account for six multiple comparisons, regarding the ROIs considered in the study. A corrected significance threshold of 0.05 was considered. Supplementary materials include the results of statistical tests per channel instead of ROIs (S2).

\section{Results}

Behavioral results

Participants have not presented any major lapses, anticipations nor no responses. They presented a total RT mean of $251.97 \mathrm{~ms}$ ( $\pm 59.73 \mathrm{~ms}$ ). Fast responses RT (Mean $=$ $210.1 \mathrm{~ms} \mathrm{SD}=3.4 \mathrm{~ms}$ ) and slow responses RT (Mean $=385.4 \mathrm{~ms}, \mathrm{SD}=13.8 \mathrm{~ms}$ ) was significantly different from one another $(t=-15.3, p<0.001)$.

fNIRS results

We lost $16.7 \%$ of participants (5 out 30 ) and $15.7 \%$ of all channels, due to bad quality signals. Approximately, we have lost 8.7 channels per participant.

SPM

For purposes of data description, we considered only channels that presented significant results for both $\mathrm{HbO}$ and $\mathrm{Hb}$ measures. We found differences between fast responses and baseline mainly on a large portion of the parietal cortex, on the motor and premotor area, inferior frontal and medial frontal cortex (Figure 4). Regarding comparisons between slow responses and baseline, we found differences only in the inferior frontal cortex (Figure 1). Comparisons between fast and slow responses showed that fast responses present more activation in the parietal areas (channels 41 and 43, P4-CP4 and P4-P6, respectively) (Figure 1).

The functional activation of the brain was found to be correlated with behavior (as measured by mean RT) only for $\mathrm{HbO}$ in a portion of the inferior parietal cortex. Table 1 shows the correlation coefficients between mean RT and $\mathrm{HbO}$ beta values for two channels. 
Table 1.

Correlations between behavioral and physiological data

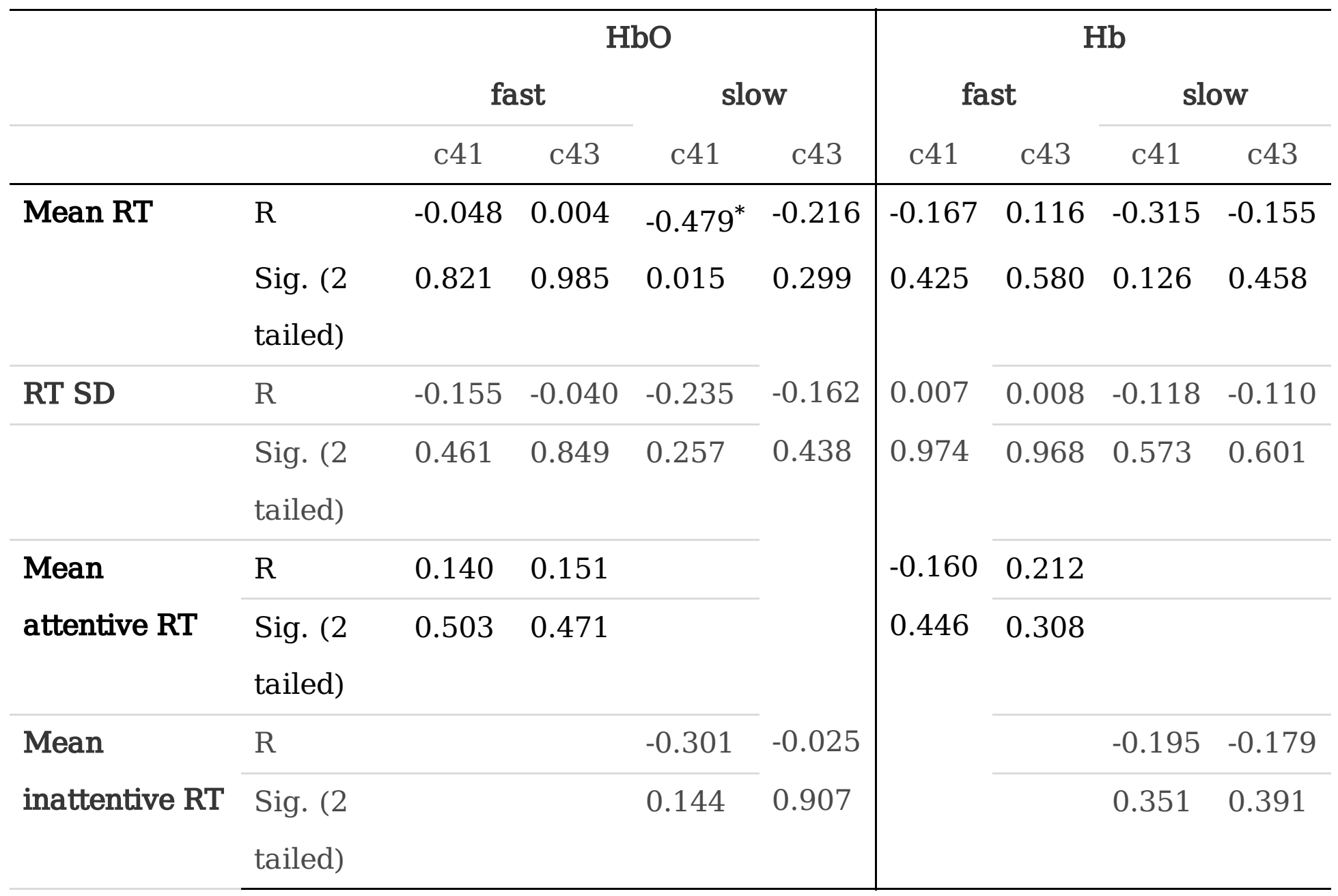

Note. Correlation between some behavioral data and beta values extracted after SPM analyses. R - Spearman correlation; Sig. - Significance; RT - Reaction Time; HbO - oxyhemoglobin; Hb deoxyhemoglobin; C - channel; SD - standard deviation.

Pre stimulus activity

We found that $\mathrm{HbO}$ concentration changes increased during fast responses and decreased during slow responses on both $\mathrm{mPFC}$ and inferior parietal cortex (Table 2). At the same time no significant differences were found on $\mathrm{Hb}$ measures between the two conditions in any ROI (Table 2), although there is a trend in the same direction of $\mathrm{HbO}$ results.

Considering the data from each channel separately, it was found that channel 2 (FPz$\mathrm{AFz}$, on mPFC ROI) presented higher $\mathrm{HbO}$ and lower $\mathrm{Hb}$ on fast responses when compared 
to slow responses. Channel 42 (P2-P4, on Inferior parietal ROI) presented higher levels of $\mathrm{HbO}$ concentration changes only during fast responses $(\mathrm{p}<0,05$ and $W$ value $=238$, see Supplementary material 3 and 4).

\section{Table 2.}

Statistics for $\mathrm{Hb}$ and $\mathrm{HbO}$ concentration changes

ROI Area Fast responses $\quad$ Slow Responses W statistic p-value

\begin{tabular}{|c|c|c|c|c|c|}
\hline & & Mean $\mathrm{HbO} \pm \mathrm{sd}(\mu \mathrm{M} \mathrm{mm})$ & \multicolumn{3}{|l|}{ Mean $\mathrm{HbO} \pm \mathrm{sd}(\mu \mathrm{M} \mathrm{mm})$} \\
\hline 1 & $\mathrm{mPFC}$ & $8.7( \pm 17.5)$ & $-4.67( \pm 20)$ & 240 & $0.037^{*}$ \\
\hline 2 & dlPFC & $0.92( \pm 27)$ & $-6.62( \pm 20.7)$ & 205 & 0.263 \\
\hline 3 & iPFC & $-6.4( \pm 34)$ & $7.4( \pm 41.0)$ & 113 & 0.191 \\
\hline 4 & Precentral & $5.0 \times 10^{-4}( \pm 14)$ & $-3.4( \pm 20.0)$ & 180 & 0.653 \\
\hline 5 & Postcentral & $-2.8( \pm 17)$ & $1.7( \pm 23)$ & 139 & 0.542 \\
\hline 6 & Sup. Parietal & $1.9( \pm 23)$ & $-0.88( \pm 17)$ & 181 & 0.634 \\
\hline 7 & Inf. Parietal & $9.9( \pm 27.6)$ & $-6.6( \pm 17)$ & 248 & $0.020^{*}$ \\
\hline 8 & Occipital & $-0.87( \pm 28)$ & $-0.54( \pm 32)$ & 157 & 0.895 \\
\hline \multicolumn{6}{|c|}{ Hb measures } \\
\hline 1 & $\mathrm{mPFC}$ & $-4.5 \times 10^{-6}\left( \pm 1.3 \times 10^{-5}\right)$ & $5.8 \times 10^{-6}\left( \pm 1.6 \times 10^{-5}\right)$ & 110 & 0.164 \\
\hline 2 & dlPFC & $-1.6 \times 10^{-6}\left( \pm 1.9 \times 10^{-5}\right)$ & $2.2 \times 10^{-6}\left( \pm 1.7 \times 10^{-5}\right)$ & 129 & 0.381 \\
\hline 3 & iPFC & $2.1 \times 10^{-6}\left( \pm 3.3 \times 10^{-5}\right)$ & $-5.4 \times 10^{-6}\left( \pm 4.2 \times 10^{-5}\right)$ & 185 & 0.560 \\
\hline 4 & Precentral & $3.3 \times 10^{-6}\left( \pm 1.4 \times 10^{-5}\right)$ & $-1.1 \times 10^{-6}\left(1.5 \times 10^{-5}\right)$ & 204 & 0.275 \\
\hline 5 & Postcentral & $7.4 \times 10^{-5}\left( \pm 1.4 \times 10^{-5}\right)$ & $-2.6 \times 10^{-6}\left( \pm 1.6 \times 10^{-5}\right)$ & 217 & 0.148 \\
\hline 6 & Sup. Parietal & $1.2 \times 10^{-6}\left( \pm 1.6 \times 10^{-5}\right)$ & $7.6 \times 10^{-7}\left( \pm 1.6 \times 10^{-5}\right)$ & 164 & 0.979 \\
\hline 7 & Inf. Parietal & $-6.1 \times 10^{-6}\left( \pm 3.2 \times 10^{-5}\right)$ & $5.1 \times 10^{-6}\left( \pm 1.3 \times 10^{-5}\right)$ & 129 & 0.381 \\
\hline 8 & Occipital & $-7.9 \times 10^{-6}\left( \pm 2.9 \times 10^{-5}\right)$ & $-4.8 \times 10^{-6}\left( \pm 4.3 \times 10^{-5}\right)$ & 129 & 0.381 \\
\hline
\end{tabular}

Note. Descriptive statistics and statistical analyses for oxyhemoglobin concentration changes on each ROI for each condition. 
ROI - Region of Interest; HbO - Oxyhemoglobin; Hb - Desoxyhemoglobin; mM-mm millimolar millimeter; sd - standard deviation; mPFC - medial Prefrontal Cortex; dlPFC dorsolateral Prefrontal Cortex; iPFC - inferior Prefrontal Cortex; Sup. - superior; Inf. Inferior.

*statistical significance $(p<0,05)$ on Wilcoxon test.

Data time course visualization

Figure 2 presents the time course of $\mathrm{HbO}$ concentration changes around stimulus presentation. Fast responses presented an increase on the levels of $\mathrm{HbO}$ changes on $\mathrm{mPFC}$ and Inferior Parietal area when compared to slow responses. The Inferior Frontal Gyrus indicated a trend in the opposite direction; although not statistically significant, in this region, $\mathrm{HbO}$ concentration changes appeared to decrease during fast responses.

Regarding the temporal dynamics of the concentration changes it is observed in Inferior Parietal ROI (Figure 2) differentiation between conditions some seconds before the stimulus presentation and a tendency to return to similar levels some seconds after the stimulus, possibly indicating that ongoing hemodynamic activity might alter the response to stimulus presentation. The pattern of activity presented in Inferior Parietal ROI can also be

observed in channel 42 individually (Figure 3). Channel 2, on the other hand, presents a rather sustained difference between conditions both before and after stimulus presentation. Additionally, it can also be observed a pattern of activity similar to a hemodynamic response in channel 3 (Figure 3) in which there is a raise on $\mathrm{HbO}$ levels only some seconds after stimulus presentation. Supplementary Material 5 presents time courses for $\mathrm{HbO}$ together with $\mathrm{Hb}$.

\section{Discussion}

The present work aimed to investigate (a) whether there are differences in the hemodynamic response between fast and slow RT; (b) differences on variation of $\mathrm{HbO}$ and $\mathrm{Hb}$ levels before stimulus onset between fast and slow RT; and (c) whether there were differences on the shape of the hemodynamic curve between fast and slow responses on different brain areas. We found differences between fast and slow RT both on the location of the hemodynamic response and on the pre stimulus activity. It was also 
found that different shapes of the time course of the hemodynamics could possibly reflect different activity patterns.

We have presented two sets of analysis. In the first of them, we conducted a GLM-based SPM. This analysis brings information on the hemodynamic response to a stimulus. Data pointed that, when compared to slow responses, fast responses present more involvement of inferior parietal areas, medial prefrontal and motor regions. These findings are analogous to (Drummond et al., 2005). Both studies found that fast responses on PVT show more activity in some right medial frontal regions, right inferior parietal lobe, and motor areas. These areas are part of a frontoparietal sustained attention network, involved in allocation of attentional resources and sustainment of attention (references).

In order to further investigate the relationship between neurophysiological and behavioral data we conducted an exploratory analysis of the correlation between RT and beta values extracted from GLM. Even though we could not find any robust correlations, our data suggest that a portion of the inferior parietal cortex (P4 and CP4 of the 10-20 International EEG System) might be related to RT variations which is in accordance with our initial location hypothesis. Similar results were found by (Bogler et al., 2014) who have demonstrated correlations between hemodynamic activity assessed by fNIRS on frontoparietal areas and RT.

In the second set of analyses, we focused on ongoing brain activity. Thus, we conducted a second set of analysis investigating differences on mean $\mathrm{Hb}$ variations before stimulus presentation. Again, there were differences on $\mathrm{Hb}$ variations between fast and slow responses on inferior parietal cortex and medial prefrontal cortex. These findings are in agreement with two fMRI studies (Bogler et al., 2014; Coste et al., 2011; Nozawa et al., 2014) showing that previous hemodynamic activity can inform further reaction time in Stroop tasks. Yet, one fNIRS study (Bogler et al., 2014) indicated negative results when attempting to predict RT through hemodynamic measures.

We have found differences between fast and slow responses both before and after stimulus onset on frontoparietal areas. These temporal characteristics, together with the localization in which they presented itself, are analogous to Dosenbach and colleagues' data (Dosenbach et al., 2007). The authors demonstrate that there are two main brain networks interplaying during cognitive tasks: a preparatory, set-maintenance activity sustained across the whole task and a responsive, adaptive activity, involved in self-monitoring and adjusting. Although our data does not bring robust evidence for the dual-network approach it is an indicative that our experimental paradigm might be a simple, less costly and effective way to test it.

Our data on pre-stimulus activity might also be in accordance with a brain state approach, as presented by (Deco et al., 2019) and (Deco et al., 2019; Ponce-Alvarez et al., n.d.). Deco et al discuss that our brain fluctuates between different mental states while attention fluctuates. Ponce-Alvarez et al. present data showing that our brain responds differently depending on which brain state is ongoing at the moment a stimulus is presented. A similar situation might have happened in our study. We hypothesize that there were two different brain states, one during which stimulus presentation could have had high probability 
of being followed by slower RT and another in which stimulus presentation could have had high probability of being followed by faster RT. In this case, good performance would not be associated by a preparatory brain activity, but to different states the brain would be found when stimuli were presented. Yet, further research is needed to better investigate the use of fNIRS to test Deco and Ponce-Alvarez brain state approach.

Our results are tightly dependable on some experimental characteristics that should be highlighted. We used a uniform randomization of the interstimulus interval which allows for some level of anticipation and preparation that might have affected behavioral performance, and consequently its neural correlates. This type of randomization allow discrimination of interstimulus interval, since the more the time have passed, more likely it was for the stimulus to appear. A randomization with an exponential distribution could lead to less predictability and possibly lead to different neurophysiological results. Another important experimental characteristic is the fact that participants received feedback on each trial, since their reaction times were shown on the screen right after each response. This fact might have led to less expressive slow responses, since they tended to improve their performance after the presentation of a high reaction time. It also might also have influenced fNIRS data, an experimental task that does not provide feedback might lead to different results.

Thus, one limitation of our study is that it does not allow for differentiation between vigilance, motor readiness and time estimation components of the physiological response. Although they might sound very similar processes, it is said that they are independent components and might have different neural correlates (Brown et al., 2011; Brunia, 1993; Hsu, 2005; Oken et al., 2006). Other disadvantages are related to fNIRS spatial and temporal specificities. In our study we have covered only the right side of the scalp, leaving aside important structures in the other hemisphere that play a role in attention. Beyond that, fNIRS is able to collect data of only superficial cortical structures and it has a spatial resolution of about two centimeters (Len-Carrin \& Len-Domnguez, 2012). This leaves out deep structures and might not differentiate different segments of proximate brain areas. It is considered an important disadvantage, since it hinders the investigation of important areas of the DMN, of the dACC/msFC network and of the al/fO network. Also, fNIRS collects data on hemodynamic activity which is too slow - happens in the time frame of seconds - when it comes to studying cognitive processes that happen in the time frame of milliseconds.

On the other hand, the fact that the hemodynamic activity is slow and that differences in performance were found both before and after the time point of interest might be an interesting solution to a different kind of problem. Since fNIRS is more tolerant to movement artifacts it has been applied on neuroscience studies in naturalistic settings (Balardin et al., 2017; Brockington et al., 2018). Yet, one challenge naturalistic studies face is the precise determination of moments in time from which to consider brain activity (Pinti et al., 2020) - in other terms, how to precisely time triggers for data analysis. Our data indicate that the differences on brain activity related to attention states can be found in two different time windows both before and after the moment of interest. This evidence might allow some flexibility on timing moments of interest when conducting naturalistic studies of attention. 


\section{Conclusion}

In summary, our study shows that there are differences of cortical hemodynamic activity on frontoparietal areas between fast and slow responses. It also shows that the data time course of this activity might reflect different brain and cognitive functions.

\section{Declarations}

\section{Ethics approval}

The present work was carried out in accordance with the Declaration of Helsinki. It was submitted to the UFABC ethical committee (CAAE: 846099318.0.0000.5594) and has been approved (number: 2.754.496).

\section{Consent to participate}

All participants signed a freely given, informed consent to participate term. This term was previously approved by the aforementioned committee.

\section{Consent to publish}

This work does not include materials from participants that require consent to publish.

\section{Author's contribution}

Author contributions included conception and study design (MGN, CEB, JRS, AFB), data collection or acquisition (MGN), statistical analysis (MGN, MS, CSFB), interpretation of results (MGN, CEB, JRS, RCM, AFB), drafting the manuscript work or revising it critically for important intellectual content (MGN, CEB, $\mathrm{RCM}, \mathrm{AFB}$ ) and approval of final version to be published and agreement to be accountable for the integrity and accuracy of all aspects of the work (All authors).

\section{Funding}

The first author of this publication received funding from Coordenação de Aperfeiçoamento de Pessoal de Nível Superior - Brasil (CAPES) - Finance Code 001.

\section{Competing interests}




\section{Availability of data and materials}

Data and code will be soon public and available at Mendeley Data (Nogueira, 2021).

\section{References}

Arsintescu, L., Mulligan, J. B., \& Flynn-Evans, E. E. (2017). Evaluation of a Psychomotor Vigilance Task for Touch Screen Devices. Human Factors, 59(4), 661-670.

Balardin, J. B., Zimeo Morais, G. A., Furucho, R. A., Trambaiolli, L., Vanzella, P., Biazoli, C., Jr, \& Sato, J. R. (2017). Imaging Brain Function with Functional Near-Infrared Spectroscopy in Unconstrained Environments. Frontiers in Human Neuroscience, 11, 258.

Bogler, C., Mehnert, J., Steinbrink, J., \& Haynes, J.-D. (2014). Decoding vigilance with NIRS. PloS One, 9(7), e101729.

Borragán, G., Guerrero-Mosquera, C., Guillaume, C., Slama, H., \& Peigneux, P. (2019). Decreased prefrontal connectivity parallels cognitive fatigue-related performance decline after sleep deprivation. An optical imaging study. Biological Psychology, 144, 115-124.

Brockington, G., Balardin, J. B., Zimeo Morais, G. A., Malheiros, A., Lent, R., Moura, L. M., \& Sato, J. R. (2018). From the Laboratory to the Classroom: The Potential of Functional Near-Infrared Spectroscopy in Educational Neuroscience. Frontiers in Psychology, 9, 1840.

Brown, H., Friston, K., \& Bestmann, S. (2011). Active inference, attention, and motor preparation. Frontiers in Psychology, 2, 218.

Brunia, C. H. (1993). Waiting in readiness: gating in attention and motor preparation. Psychophysiology, 30(4), 327-339.

Carlisi, C. O., Norman, L., Murphy, C. M., Christakou, A., Chantiluke, K., Giampietro, V., Simmons, A., Brammer, M., Murphy, D. G., MRC AIMS Consortium, Mataix-Cols, D., \& Rubia, K. (2017). Disorder-Specific and Shared Brain Abnormalities During Vigilance in Autism and Obsessive-Compulsive Disorder. Biological Psychiatry. Cognitive Neuroscience and Neuroimaging, 2(8), 644-654.

Cope, M., \& Delpy, D. T. (1988). System for long-term measurement of cerebral blood and tissue oxygenation on newborn infants by near infra-red transillumination. Medical \& Biological Engineering \& Computing, 26(3), 289-294. 
Coste, C. P., Sadaghiani, S., Friston, K. J., \& Kleinschmidt, A. (2011). Ongoing Brain Activity Fluctuations Directly Account for Intertrial and Indirectly for Intersubject Variability in Stroop Task Performance. In Cerebral Cortex (Vol. 21, Issue 11, pp. 2612-2619). https://doi.org/10.1093/cercor/bhr050

Deco, G., Cruzat, J., Cabral, J., Tagliazucchi, E., Laufs, H., Logothetis, N. K., \& Kringelbach, M. L. (2019). Awakening: Predicting external stimulation to force transitions between different brain states. Proceedings of the National Academy of Sciences of the United States of America, 116(36), 1808818097.

Dinges, D. F., \& Powell, J. W. (1985). Microcomputer analyses of performance on a portable, simple visual RT task during sustained operations. In Behavior Research Methods, Instruments, \& Computers (Vol. 17, Issue 6, pp. 652-655). https://doi.org/10.3758/bf03200977

Dosenbach, N. U. F., Fair, D. A., Miezin, F. M., Cohen, A. L., Wenger, K. K., Dosenbach, R. A. T., Fox, M. D., Snyder, A. Z., Vincent, J. L., Raichle, M. E., Schlaggar, B. L., \& Petersen, S. E. (2007). Distinct brain networks for adaptive and stable task control in humans. Proceedings of the National Academy of Sciences of the United States of America, 104(26), 11073-11078.

Drummond, S. P. A., Bischoff-Grethe, A., Dinges, D. F., Ayalon, L., Mednick, S. C., \& Meloy, M. J. (2005). The neural basis of the psychomotor vigilance task. Sleep, 28(9), 1059-1068.

Fortenbaugh, F. C., DeGutis, J., \& Esterman, M. (2017). Recent theoretical, neural, and clinical advances in sustained attention research. Annals of the New York Academy of Sciences, 1396(1), 70-91.

Hall, R. W., Huitt, T. W., Thapa, R., Williams, D. K., Anand, K. J. S., \& Garcia-Rill, E. (2008). Long-term deficits of preterm birth: evidence for arousal and attentional disturbances. Clinical Neurophysiology: Official Journal of the International Federation of Clinical Neurophysiology, 119(6), 1281-1291.

Harrivel, A. R., Weissman, D. H., Noll, D. C., \& Peltier, S. J. (2013). Monitoring attentional state with fNIRS. Frontiers in Human Neuroscience, 7, 861.

Hinds, O., Thompson, T. W., Ghosh, S., Yoo, J. J., Whitfield-Gabrieli, S., Triantafyllou, C., \& Gabrieli, J. D. E. (2013). Roles of default-mode network and supplementary motor area in human vigilance performance: evidence from real-time fMRI. Journal of Neurophysiology, 109(5), 1250-1258.

Hiroyasu, T., Fukushima, A., \& Yokouchi, H. (2012). Differences in blood flow between auditory and visual stimuli in the Psychomotor Vigilance Task and GO/NOGO Task. Conference Proceedings: ... Annual International Conference of the IEEE Engineering in Medicine and Biology Society. IEEE Engineering in Medicine and Biology Society. Conference, 2012, 1466-1469.

Hosomi, F., Yanagi, M., Kawakubo, Y., Tsujii, N., Ozaki, S., \& Shirakawa, O. (2019). Capturing spontaneous activity in the medial prefrontal cortex using near-infrared spectroscopy and its application to schizophrenia. Scientific Reports, 9(1), 5283. 
Hsu, Y.-F. (2005). On measuring the minimum detection time: a simple reaction time study in the time estimation paradigm. The British Journal of Mathematical and Statistical Psychology, 58(Pt 2), 259-284.

Huppert, T. J., Diamond, S. G., Franceschini, M. A., \& Boas, D. A. (2009). HomER: a review of time-series analysis methods for near-infrared spectroscopy of the brain. Applied Optics, 48(10), D280-D298.

Jaipurkar, R., Mahapatra, S. S., Bobdey, S., \& Banerji, C. (2019). Work-rest pattern, alertness and performance assessment among naval personnel deployed at sea: A cross sectional study. Armed Forces Medical Journal, India, 75(2), 158-163.

Khitrov, M. Y., Laxminarayan, S., Thorsley, D., Ramakrishnan, S., Rajaraman, S., Wesensten, N. J., \& Reifman, J. (2014). PC-PVT: a platform for psychomotor vigilance task testing, analysis, and prediction. Behavior Research Methods, 46(1), 140-147.

Klem, G. H., Lüders, H. O., Jasper, H. H., \& Elger, C. (1999). The ten-twenty electrode system of the International Federation. The International Federation of Clinical Neurophysiology.

Electroencephalography and Clinical Neurophysiology. Supplement, 52, 3-6.

Langner, R., \& Eickhoff, S. B. (2013). Sustaining attention to simple tasks: A meta-analytic review of the neural mechanisms of vigilant attention. In Psychological Bulletin (Vol. 139, Issue 4, pp. 870-900). https://doi.org/10.1037/a0030694

Lawrence, N. S., Ross, T. J., Hoffmann, R., Garavan, H., \& Stein, E. A. (2003). Multiple neuronal networks mediate sustained attention. Journal of Cognitive Neuroscience, 15(7), 1028-1038.

Len-Carrin, J., \& Len-Domnguez, U. (2012). Functional Near-Infrared Spectroscopy (fNIRS): Principles and Neuroscientific Applications. In Neuroimaging - Methods. https://doi.org/10.5772/23146

McHill, A. W., \& Wright, K. P., Jr. (2019). Cognitive Impairments during the Transition to Working at Night and on Subsequent Night Shifts. Journal of Biological Rhythms, 34(4), 432-446.

Morais, G. A. Z., Balardin, J. B., \& Sato, J. R. (2018). fNIRS Optodes' Location Decider (fOLD): a toolbox for probe arrangement guided by brain regions-of-interest. In Scientific Reports (Vol. 8, Issue 1). https://doi.org/10.1038/s41598-018-21716-z

Nogueira, Mateus (2021), "PVT_fNIRS_UFABC_01”, Mendeley Data, V1, doi: 10.17632/3xg8c9yfg6.1

Nordin, L. E., Möller, M. C., Julin, P., Bartfai, A., Hashim, F., \& Li, T.-Q. (2016). Post mTBI fatigue is associated with abnormal brain functional connectivity. In Scientific Reports (Vol. 6, Issue 1). https://doi.org/10.1038/srep21183

Nozawa, T., Sugiura, M., Yokoyama, R., Ihara, M., Kotozaki, Y., Miyauchi, C. M., Kanno, A., \& Kawashima, R. (2014). Ongoing activity in temporally coherent networks predicts intra-subject fluctuation of response time to sporadic executive control demands. PloS One, 9(6), e99166. 
Oken, B. S., Salinsky, M. C., \& Elsas, S. M. (2006). Vigilance, alertness, or sustained attention: physiological basis and measurement. Clinical Neurophysiology: Official Journal of the International Federation of Clinical Neurophysiology, 117(9), 1885-1901.

Pinti, P., Tachtsidis, I., Hamilton, A., Hirsch, J., Aichelburg, C., Gilbert, S., \& Burgess, P. W. (2020). The present and future use of functional near-infrared spectroscopy (fNIRS) for cognitive neuroscience. Annals of the New York Academy of Sciences, 1464(1), 5-29.

Ponce-Alvarez, A., Mochol, G., Hermoso-Mendizabal, A., de la Rocha, J., \& Deco, G. (n.d.). Cortical state transitions and stimulus response evolve along stiff and sloppy parameter dimensions, respectively. https://doi.org/10.1101/839365

Raichle, M. E., \& Snyder, A. Z. (2007). A default mode of brain function: A brief history of an evolving idea. In Neurolmage (Vol. 37, Issue 4, pp. 1083-1090). https://doi.org/10.1016/j.neuroimage.2007.02.041

Rodrigues, J. de S., de Souza Rodrigues, J., Ribeiro, F. L., Sato, J. R., Mesquita, R. C., \& Júnior, C. E. B. (2019). Identifying individuals using fNIRS-based cortical connectomes. In Biomedical Optics Express (Vol. 10, Issue 6, p. 2889). https://doi.org/10.1364/boe.10.002889

Sturm, W., \& Willmes, K. (2001). On the functional neuroanatomy of intrinsic and phasic alertness. Neurolmage, 14(1 Pt 2), S76-S84.

Yu, C., \& Smith, L. B. (2016). The Social Origins of Sustained Attention in One-Year-Old Human Infants. Current Biology: CB, 26(9), 1235-1240.

\section{Figures}




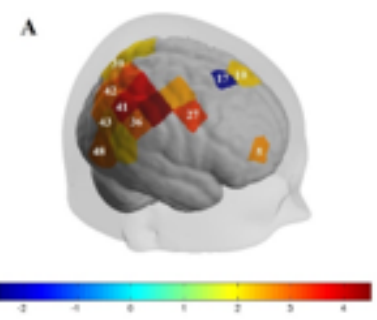

Fast $>$ Mean

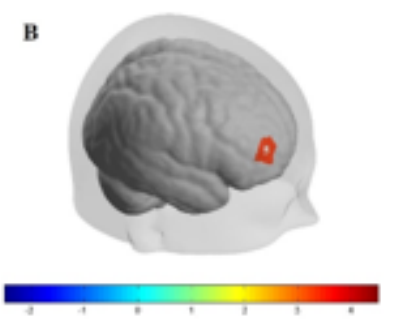

Slow $>$ Mean
C

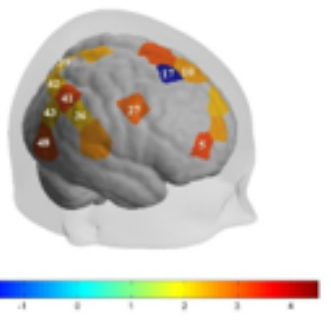

Fast $>$ Mean

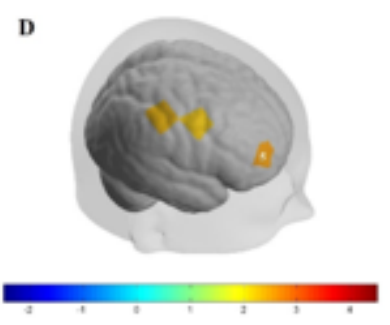

Slow $>$ Mean

F.

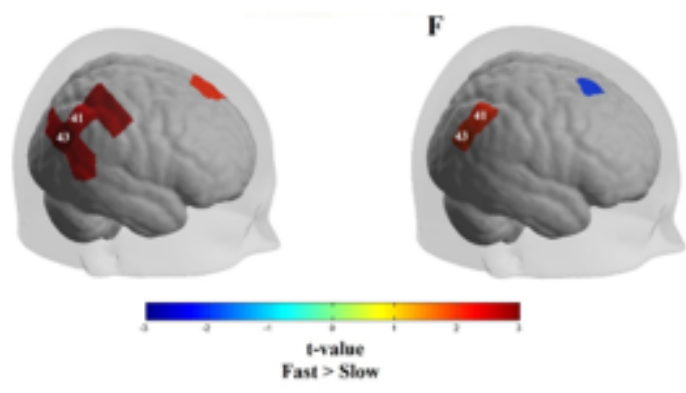

\section{Figure 1}

SPM comparing fast or slow responses with general activity during the experiment, and comparing fast with slow activity. Colored areas represent channels that have shown significant differences $(p<0.05)$. Colors represent t-values for each channel. Some relevant channels are numbered.
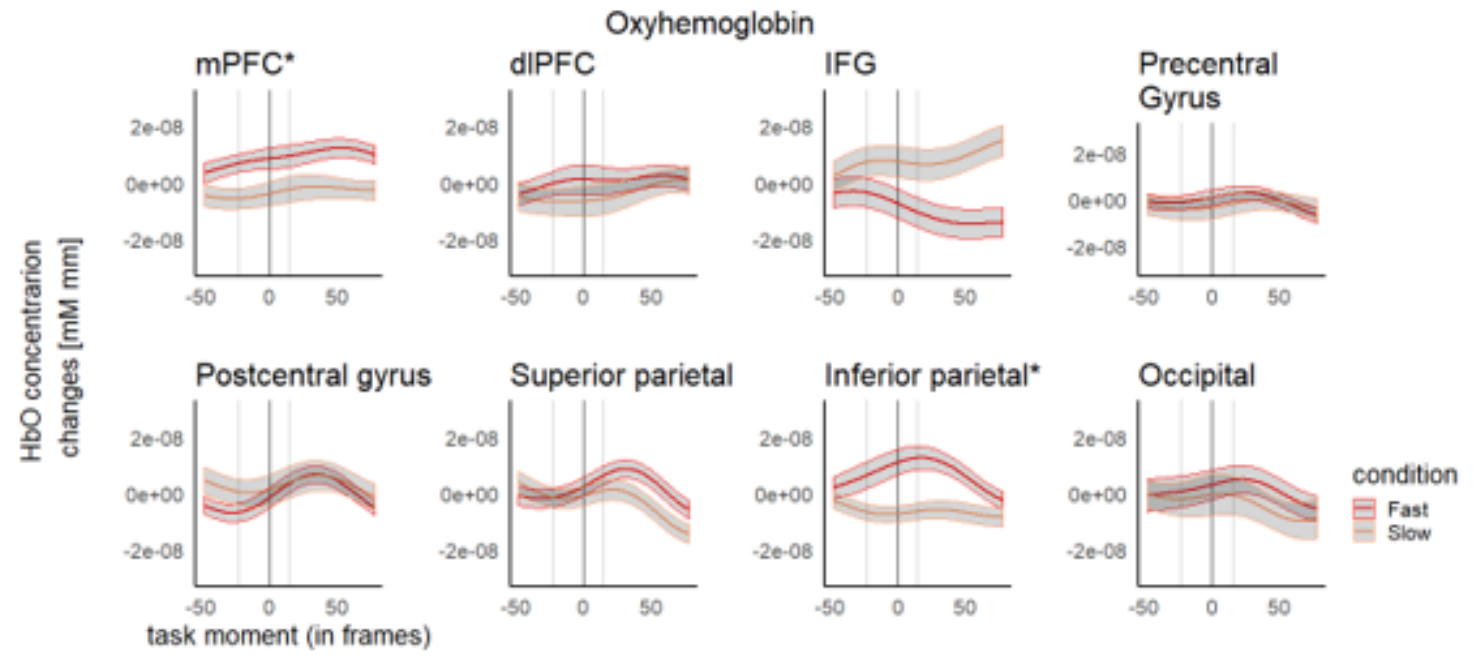

task moment [frames]

Figure 2 
$\mathrm{HbO}$ time courses. Note. Averaged time course across all participants of $\mathrm{HbO}$ concentration changes between fast and slow conditions. The count of 50 frames on y axis corresponds to approximately $6 \mathrm{~s}$. Black vertical line represents stimulus presentation; gray vertical lines circumscribe the period from which data was considered for statistical tests. ${ }^{*}$ ROls that presented statistically significant differences between conditions on Wilcoxon test ( $p$-value $<0,05$ ).

Channels

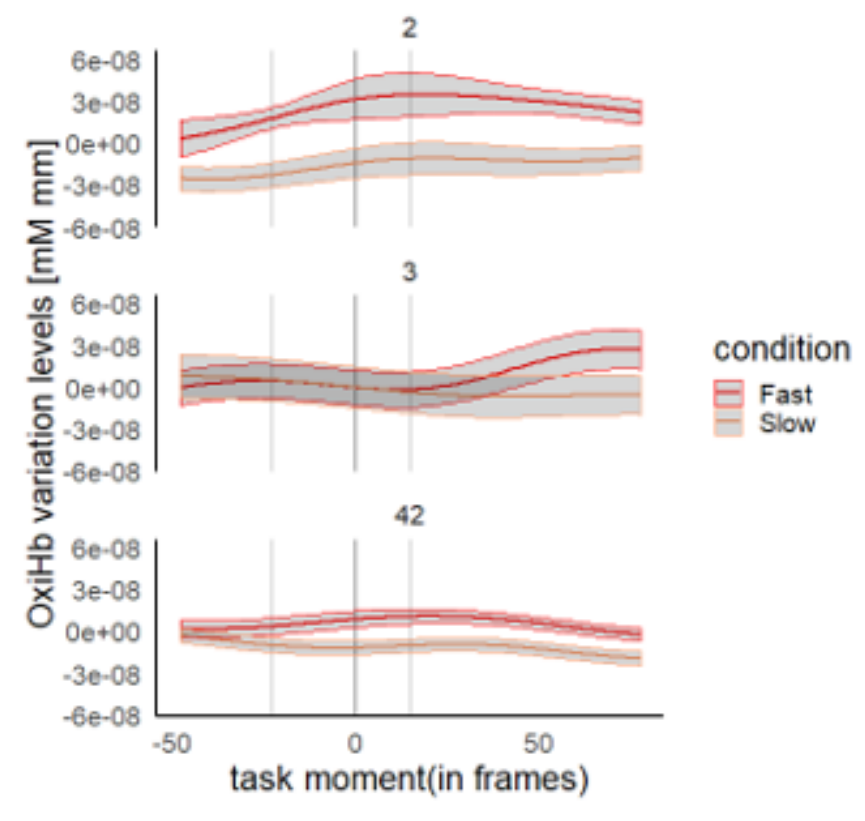

\section{Figure 3}

Time courses of three specific channels. Note. Averaged time course across all participants of $\mathrm{HbO}$ concentration changes between attentive and inattentive conditions in channel 2 (FpZ-AfZ), channel 3 (Af4-Fp2), and channel 42 (P2-P4). Fifty frames correspond to approximately 6s. The black vertical line represents stimulus presentation; gray vertical lines circumscribe the period from which data was considered for statistical tests.

\section{Supplementary Files}

This is a list of supplementary files associated with this preprint. Click to download.

- SupplementaryMaterials.docx

- Supplementarymaterial5Timecourses.jpg 